Supplemental Information for

\title{
Reverse Photochromic Behavior of Iron-Magnesium Complex
}

\author{
Minoru Kobayashi, ${ }^{\dagger, \ddagger, \star}$ Akito Takashima, ${ }^{\perp}$ Tomohiko Ishii, ${ }^{\perp}$ Hiroshi Naka, ${ }^{\S}$ Masanobu \\ Uchiyama\#, and Kentaro Yamaguchi, ${ }^{\dagger, \ddagger, *}$ \\ Faculty of Pharmaceutical Sciences at Kagawa Campus, Tokushima Bunri University, 1314-1 Shido, \\ Sanuki, Kagawa 796-2193, Japan, CREST, Japan Science and Technology Agency (JST), Faculty of \\ Engineering, Kagawa University, Takamatsu 761-0396, Japan, Graduate School of Pharmaceutical \\ Sciences, Tohoku University, Aobayama, Aoba-ku, Sendai 980-8578, Japan, and Advanced Elements \\ Chemistry Laboratory, The Institute of Physical and Chemical Research, RIKEN, 2-1 Hirosawa, \\ Wako-shi, Saitama 351-0198, Japan
}

General Comments. All experiments were carried out under dried nitrogen using standard Schlenk techniques. Methylmagnesium bromide and anhydrous tetrahydrofuran were purchased from Kanto Chemical Co. Ltd., Wako Pure Chemical Industries Ltd., and were used without further purification. Iron(II) chloride was purchased from Wako Pure Chemical Industries Ltd., and was dried under vacuum over $5 \mathrm{~h}$. The UV irradiations were carried out using the light of a $150 \mathrm{~W}$ tungsten-xenon source equipped with the cutoff filters to pass the $365 \mathrm{~nm}$ wavelength.

Preparation of (thf) ${ }_{4} \mathbf{M g}\left(\mu-\mathrm{Br}_{2} \mathrm{FeBr}_{2}(\mathbf{1})\right.$. To a heterogeneous solution of iron(II) chloride $(2.3 \mathrm{~g}, 18 \mathrm{mmol})$ in tetrahydrofuran $(100 \mathrm{~mL})$ was added methylmagnesium bromide (89 mmol) at $-78{ }^{\circ} \mathrm{C}$, and the mixture was stirred at room temperature for $12 \mathrm{~h}$. The mixture was concentrated and washed three times with hexane $(50 \mathrm{~mL})$. The precipitant was collected and recrystallized with tetrahydrofuran to exclude the excess amount of salts. The residual filtrate was concentrated and purified by the recrystallization with dichloromethane $(20 \mathrm{~mL})$ to give 1 as the air-sensitive crystals $(3.7 \mathrm{~g}, 30 \%) . \mathrm{C}_{16} \mathrm{H}_{32} \mathrm{O}_{4} \mathrm{Br}_{4} \mathrm{FeMg}$, colorless block, monoclinic, space group $P 2_{1} / c, a=16.5172(16), b=9.7901(9), c=16.2392(15) \AA, \beta$ $=114.2880(10)^{\circ}, V=2393.5(4) \AA^{3}, Z=4, D_{\text {calcd }}=1.910 \mathrm{~g} / \mathrm{cm}^{3}$. Data collected on a Bruker $\mathrm{CCD} /$ Smart 1000 diffractometer with graphite-monochromated Mo K $\alpha$ radiation $(\lambda=$ $0.71073 \AA$ ) at $\mathrm{T}=100 \mathrm{~K}, 2 \theta_{\max }=55.0^{\circ}$. The structures were solved by direct methods and refined with full-matrix least-squares procedures, $R_{1}=0.0446$ and $w R_{2}=0.1289,13926$ reflections measured, 5403 unique $\left(R_{\text {int }}=0.0395\right)$ which were used in all calculations.

ESR. JEOL JES-FA100 spectrometer was used for the measurement of X-band ESR spectra, which were refered to the $\mathrm{Mn}^{2+} / \mathrm{MnO}$ marker. Complex 1 was dissolved in THF (3.68 $\mathrm{mM})$, and measured ESR at $77 \mathrm{~K}$. After the measurement, the sample was irradiated at room temperature for 5 minutes. The solution color was changed from yellow to colorless, then measured ESR again at $77 \mathrm{~K}$. Conditions: sweep time, $15 \mathrm{~min}$; center field, $280 \mathrm{mT}$; sweep width, $250 \mathrm{mT}$; modulation frequency, $100 \mathrm{kHz}$; modulation width, $0.6 \mathrm{mT}$; amplitude, 300; time constant, $0.03 \mathrm{sec}$; microwave frequency, $9115.340 \mathrm{MHz}$; microwave power, $0.998 \mathrm{~mW}$. 
UV-Vis Spectroscopy. UV-Vis spectra were acquired on a JASCO V-560 spectrometer fitted with an ETC-505T Peltier temperature control unit. The measurements were started after the irradiation to the THF solution of $1(0.48 \mathrm{mM})$. The kinetics were investigated from the absorption change at $365 \mathrm{~nm}$ and fit of the data to second-order kinetics $\left(\mathrm{r}^{2}=\right.$ 0.9954). Kinetic analysis of the reaction points to a second-order reaction. The rate constants $\left(k_{\mathrm{obs}}\right)$ was observed to be $3.38 \times 10^{3} \mathrm{M}^{-1} \mathrm{~s}^{-1}$.

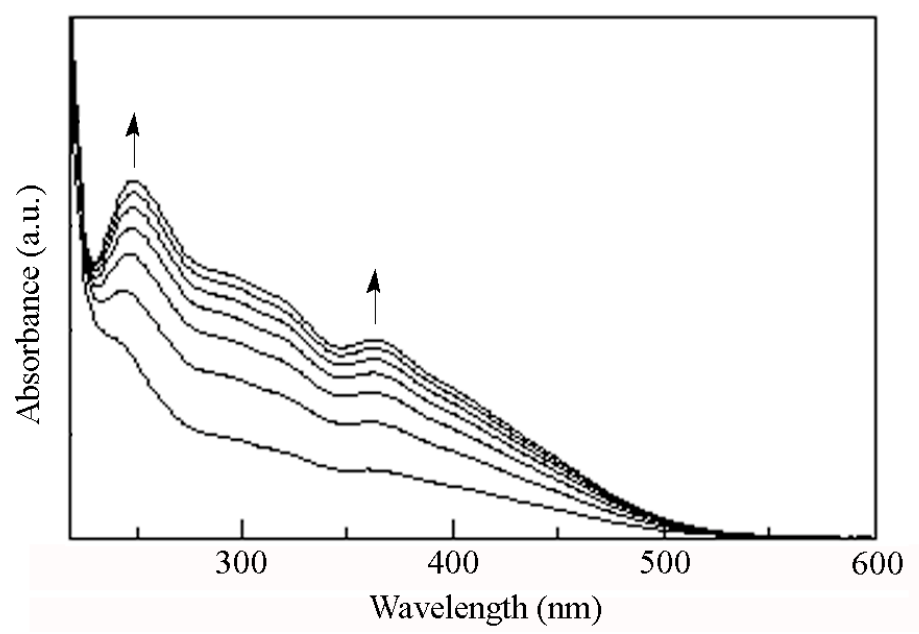

Figure S1. Time response of the UV-vis spectra of 1, measured at intervals of 30 minutes after the dissolvement in THF.

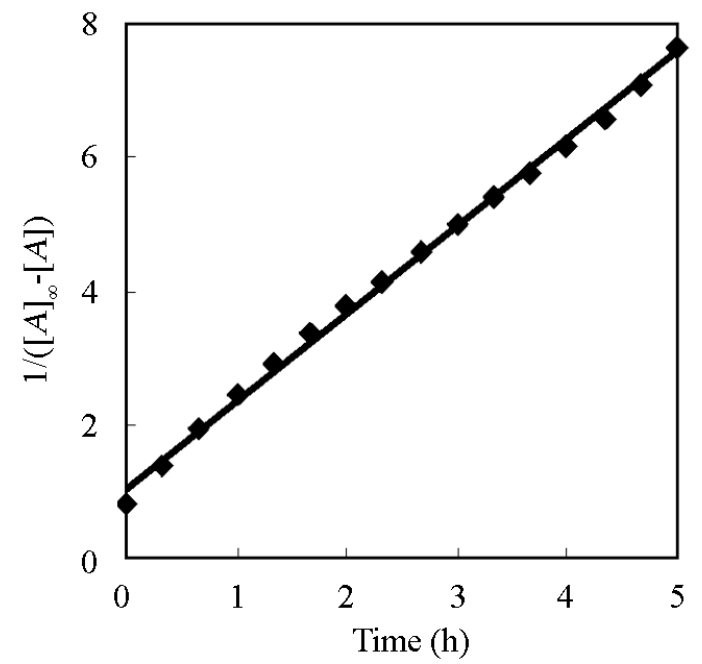

Figure S2. Plot of $1 /\left([A]_{\infty}-[A]\right)$ vs time. The solid line shows the fitting based on a second order reaction $\left(r^{2}=0.9954\right)$. 
Cyclic voltammetry. Cyclic voltammetry (CV) was recorded by using a Hokuto Denko HZ-5000 apparatus. Measurements were performed at $100 \mathrm{mV} \mathrm{s}^{-1}$ in tetrahydrofuran $(0.1$ $\mathrm{mM}$ ), using a Pt counter, working electrode, and a $\mathrm{Ag} / \mathrm{AgCl}$ reference electrode with [" $\left.\mathrm{Bu}_{4} \mathrm{P}\right]\left[\mathrm{PF}_{6}\right](0.1 \mathrm{M})$ as an electrolyte. Benchmarked redox couple with ferrocene(II)/(III) was observed at $E_{1 / 2}=188 \mathrm{mV}$ and $\Delta E_{\mathrm{p}}=98 \mathrm{mV}$.

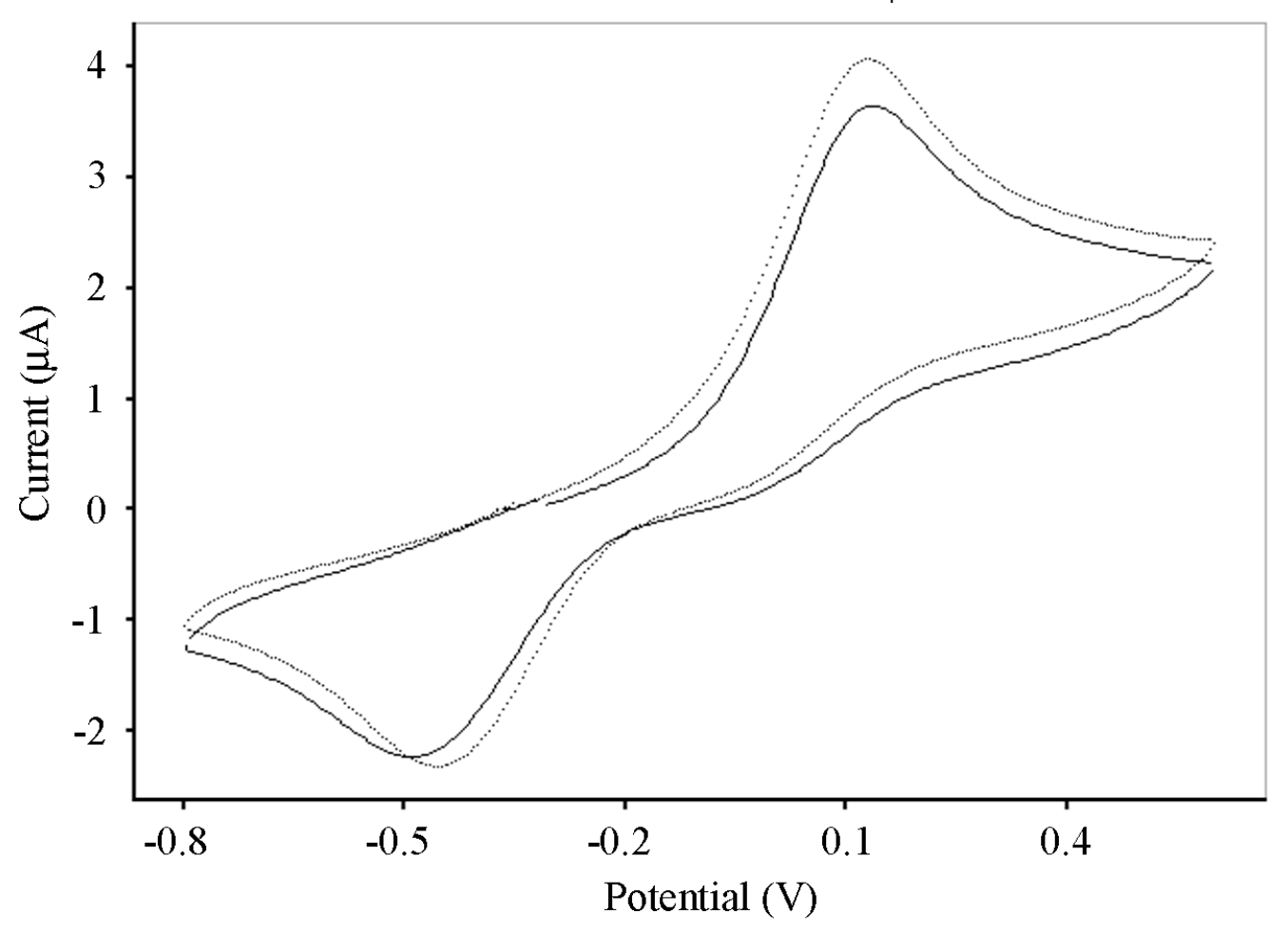

Figure S3. Cyclic voltammograms of $1(0.1 \mathrm{mM})$ in tetrahydrofuran/[ $\left.{ }^{\mathrm{n}} \mathrm{Bu}_{4} \mathrm{P}\right] \mathrm{PF}{ }_{6}$ $(0.1 \mathrm{M})$ at $V=100 \mathrm{mV} \mathrm{s}^{-1}$ (a) before UV irradiation (solid line), (b) after UV irradiation (dashed line).

Magnetic measurements (Evans method). A solution of 1 in THF (4.83 mM) placed in a $5 \mathrm{~mm}$ NMR tube, while pure THF- $d^{8}$ was placed in a capillary tube. Calculations of magnetic moments were based on the difference in the chemical shift observed for the residual THF signal in neat solvent and in the solution containing the paramagnetic species.

$$
\chi_{\mathrm{g}}=-3 \Delta \mathrm{f} / 4 \pi \mathrm{fm}+\chi_{0}+\chi_{0}\left(\delta_{0}-\delta_{\mathrm{s}}\right) / \mathrm{m}
$$




\section{Computational Details.}

Gaussian 03. All calculations were carried out with a Gaussian 03 (G03) program package $^{1}$ using the hybrid density functional method based on Becke's three-parameter exchange function and the Lee-Yang-Parr nonlocal correlation functional (B3LYP). ${ }^{2}$ We used TZVP 3 basis set for iron ${ }^{4}$ and bromine atoms, $6-31+\mathrm{G}^{*}$ for the other atoms. Geometry optimization, vibrational analysis and time-dependent DFT (TD-DFT) ${ }^{5}$ calculation were performed at the same level.

We employed $\left(\mathrm{Me}_{2} \mathrm{O}\right)_{4} \mathrm{Mg}(\mu-\mathrm{Br})_{2} \mathrm{MgBr}_{2}$ (1a) as a chemical model for (thf $)_{4} \mathrm{Mg}(\mu$ $\mathrm{Br})_{2} \mathrm{MgBr}_{2}$ (1). This model was optimized without any symmetry assumptions and characterized by vibrational analysis to confirm its identity as an energy minimum.

The quintet state of 1a was found to be reasonably lowest in energy compared to other spin states. The low-lying 30 excited states of 1a were computed using TD-DFT methodology, and no significant oscillator strength $(f)$ has been detected here (wavelengths from $3454 \mathrm{~nm}$ to $267 \mathrm{~nm}$ ).

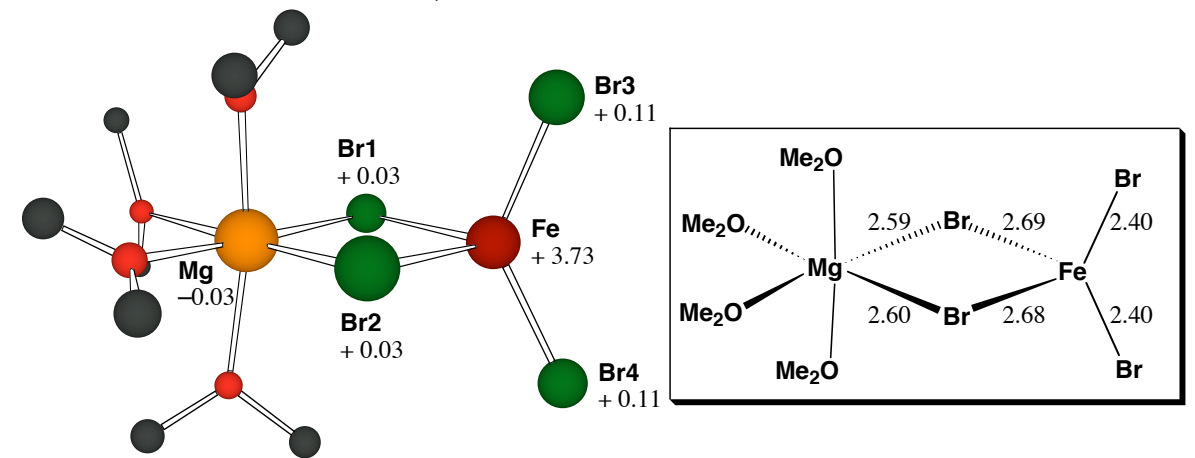

Figure S4. An optimized structure of $\left(\mathrm{Me}_{2} \mathrm{O}\right)_{4} \mathrm{Mg}\left(\mu-\mathrm{Br}_{2} \mathrm{MgBr}_{2}\right.$ (1a). Hydrogen atoms are omitted for clarity. Spin densities larger than 0.01 are marked on each atom (left). Calculated bond lengths are shown in angstroms (right).

${ }^{1}$ Frish, M. J. et al. Gaussian 03, revision c.01; Gaussian, Inc.; Wallingford, CT, 2004.

2 (a) Becke, A. D. Phys. Rev. 1998, A38, 3098-3100. (b) Becke, A. D. J. Chem. Phys. 1993, 98, 1372-1377. (c) Becke, A. D. J. Chem. Phys. 1993, 98, 5648-5652. (d) Lee, C.; Yang, W.; Parr, R. G. Phys. Rev. 1998, B37, 785-788.

${ }^{3}$ Godbout, N.; Salahub, D. R.; Andzelm, J.; Wimmer, E. Can. J. Chem. 1992, 70, 560.

${ }^{4}$ A recent example: Irigoras, A; Mercero, J. M.; Silanes, I; Uglade, J. M. J. Am. Chem. Soc. 2001, 123, 5040-5043.

${ }^{5}$ Runge, E.; Gross, E. K. U. Phys. Rev. Lett. 1984, 52, 997-1000. 
Cartesian coordinates of $1 \mathrm{a}$.

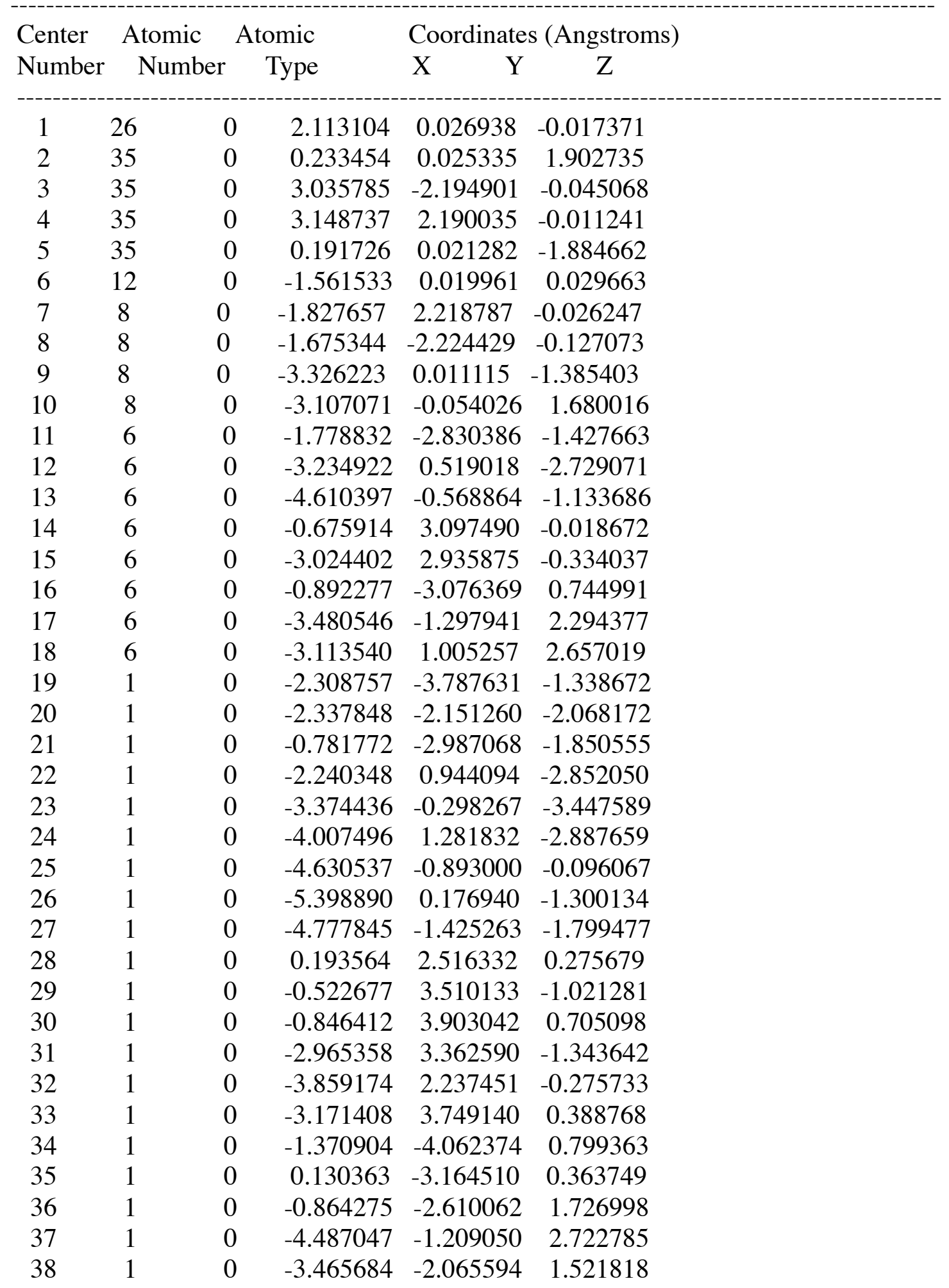




$\begin{array}{lllrrr}39 & 1 & 0 & -2.765679 & -1.556460 & 3.084647 \\ 40 & 1 & 0 & -2.784505 & 1.913729 & 2.158451 \\ 41 & 1 & 0 & -4.131100 & 1.128501 & 3.048334 \\ 42 & 1 & 0 & -2.418344 & 0.769510 & 3.469441\end{array}$

\section{TD-DFT Calculation of 1a.}

$\mathrm{TD}=(\mathrm{NSTATES}=30)$

Excitation energies and oscillator strengths:

Excited State 1: ?Spin -A $0.3589 \mathrm{eV} 3454.69 \mathrm{~nm} \mathrm{f}=0.0000$

Excited State 2: ?Spin -A $\quad 0.5283 \mathrm{eV} 2346.66 \mathrm{~nm} \quad \mathrm{f}=0.0002$

Excited State 3: ?Spin -A $0.5917 \mathrm{eV} 2095.39 \mathrm{~nm} \mathrm{f}=0.0000$

Excited State 4: ?Spin -A $0.6433 \mathrm{eV} 1927.19 \mathrm{~nm} \mathrm{f}=0.0000$

Excited State 5: ?Spin -A $3.6761 \mathrm{eV} 337.27 \mathrm{~nm} \mathrm{f}=0.0002$

Excited State 6: ?Spin -A $3.6822 \mathrm{eV} 336.71 \mathrm{~nm} \mathrm{f}=0.0003$

Excited State 7: ?Spin -A $3.7229 \mathrm{eV} 333.03 \mathrm{~nm} \mathrm{f}=0.0105$

Excited State 8: ?Spin -A $3.7641 \mathrm{eV} 329.39 \mathrm{~nm} \mathrm{f}=0.0020$

Excited State 9: ?Spin -A $3.8397 \mathrm{eV} 322.90 \mathrm{~nm} \mathrm{f}=0.0020$

Excited State 10: ?Spin -A $3.8495 \mathrm{eV} 322.08 \mathrm{~nm}$ f=0.0016

Excited State 11: ?Spin -A $3.8797 \mathrm{eV} 319.57 \mathrm{~nm} \mathrm{f}=0.0023$

Excited State 12: ?Spin -A $3.8859 \mathrm{eV} 319.06 \mathrm{~nm} \mathrm{f}=0.0036$

Excited State 13: ?Spin -A $3.9035 \mathrm{eV} 317.63 \mathrm{~nm}$ f $=0.0001$

Excited State 14: ?Spin -A $3.9947 \mathrm{eV} 310.37 \mathrm{~nm} \mathrm{f}=0.0073$

Excited State 15: ?Spin -A $4.0603 \mathrm{eV} 305.36 \mathrm{~nm} \mathrm{f}=0.0237$

Excited State 16: ?Spin -A $4.0812 \mathrm{eV} 303.79 \mathrm{~nm} \mathrm{f}=0.0001$

Excited State 17: ?Spin -A $\quad 4.1270 \mathrm{eV} 300.42 \mathrm{~nm} \mathrm{f}=0.0183$

Excited State 18: ?Spin -A $4.2101 \mathrm{eV} 294.49 \mathrm{~nm} \mathrm{f}=0.0003$

Excited State 19: ?Spin -A $4.3097 \mathrm{eV} 287.69 \mathrm{~nm} \mathrm{f}=0.0001$

Excited State 20: ?Spin -A $4.3764 \mathrm{eV} 283.30 \mathrm{~nm} \mathrm{f}=0.0202$

Excited State 21: ?Spin -A $\quad 4.3777 \mathrm{eV} 283.22 \mathrm{~nm} \mathrm{f}=0.0057$

Excited State 22: ?Spin -A $4.4100 \mathrm{eV} 281.14 \mathrm{~nm} \mathrm{f}=0.0001$

Excited State 23: ?Spin -A $4.4438 \mathrm{eV} 279.00 \mathrm{~nm} \mathrm{f}=0.0004$

Excited State 24: ?Spin -A $4.4563 \mathrm{eV} 278.22 \mathrm{~nm} \mathrm{f}=0.0033$

Excited State 25: ?Spin -A $4.4750 \mathrm{eV} \quad 277.06 \mathrm{~nm} \mathrm{f}=0.0013$

Excited State 26: ?Spin -A $4.5289 \mathrm{eV} 273.76 \mathrm{~nm} \mathrm{f}=0.0016$

Excited State 27: ?Spin -A $4.5455 \mathrm{eV} 272.76 \mathrm{~nm} \mathrm{f}=0.0022$

Excited State 28: ?Spin -A $\quad 4.5789 \mathrm{eV} 270.77 \mathrm{~nm} \mathrm{f}=0.0003$

Excited State 29: ?Spin -A $\quad 4.5945 \mathrm{eV} 269.85 \mathrm{~nm} \mathrm{f}=0.0016$

Excited State 30: ?Spin -A $\quad 4.6397 \mathrm{eV} 267.23 \mathrm{~nm}$ f=0.0002 
DV-X $\boldsymbol{\alpha}$. In order to compare the electronic structures between monomer and dimer structures, a new expected cluster model based on the dimer structure should be required. The cluster model $\left(\mathrm{FeBr}_{4}\right.$ (thf)) discussed in this study has been built semiempirically, as following:

1. The structurally-similar molecule of the expected dimer structure, tetramagnesium molecule was prepared as a based cluster model. ${ }^{1}$

2. Substitute iron and bromine atoms for corresponding magnesium and chlorine atom positions, respectively.

3. Revise the bond lengths among the iron, magnesium and brome atoms according to the statistical factor $(\mathrm{M}-\mathrm{Br} / \mathrm{M}-\mathrm{Cl}=1.06)$, and extend the cluster size similarly. The statistical factor can be found by the software "MOGUL" (http://www.ccdc.cam.ac.uk/products/csd_system/mogul/).

The cluster model extracted a part of the $\mathrm{FeBr}_{4}$ (thf) from the revised structure was employed for the molecular orbital calculation, because the cluster model is large enough to discuss the energy level structures of the $3 d$-electron orbitals of the iron atoms. The nonrelativistic DV-Xalpha calculations of $\mathbf{1}$ and the cluster model were performed with the Slater exchange parameter, alpha, of 0.7 for all atoms and with 29000 and 9000 DV sampling points, respectively, which provided a precision of less than $0.1 \mathrm{eV}$ for valence electron energy eigenvalues. We employed the basis functions of the iron and bromine atoms up to $4 p$ orbitals. The calculations were carried out self-consistently until the difference in orbital populations between the initial and final states of the iteration was less than 0.01 electron/orbital. The computational details of the non-relativistic (DV-HFS) method used in the present work have been described elsewhere. ${ }^{2}$

\section{Reference}

1. $\mathrm{C}_{28} \mathrm{H}_{58} \mathrm{Cl}_{6} \mathrm{Mg}_{4} \mathrm{O}_{6}$; Diethyl-hexachloro-hexa(tetrahydrofuran)-tetramagnesium, J. Toney and G. D. Stucky, J. Organomet. Chem., 1971, 28, 5.

2. H. Adachi, M. Tsukada, and C. Satoko, J. Phys. Soc. Jpn., 1978, 45, 875. 


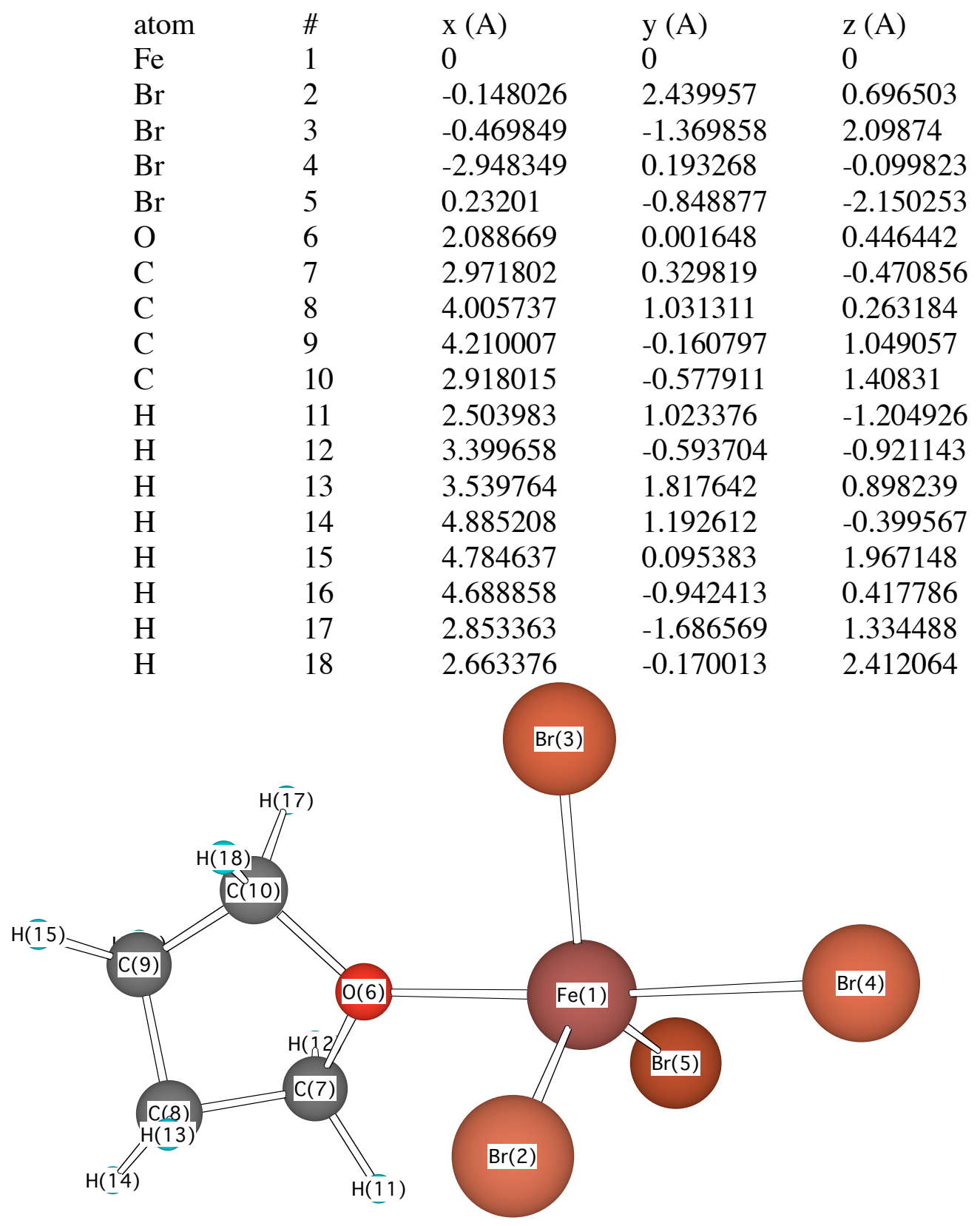

